\title{
Review Paper on Air Pollution Monitoring system
}

\author{
Snehal Sirsikar ${ }^{1}$, Priya Karemore ${ }^{2}$ \\ P.G Student, Department Of Computer Technology, Priyadarshini College of Engineering, Nagpur, India ${ }^{1}$
}

Assistant Professor, Department Of Computer Technology, Priyadarshini College of Engineering, Nagpur, India ${ }^{2}$

\begin{abstract}
Air pollution monitoring is though old but very useful concept in day to day life. Air pollution monitoring start from traditional way to the most sophisticated computer has been used to monitor the air quality, however the fresh air is necessary for all human being, for that various technology has been used and some of this technology is really useful in order to provide a real time air quality data. Aim of this paper is to highlight some technology which is used for air pollution monitoring and how effective of these technologies are and identify the important research in this important area.
\end{abstract}

Keywords: Wireless sensor network, Air quality, pollution, real time monitoring.

\section{INTRODUCTION}

Environmental monitoring is a systematic approach for observing and studying the condition of environment. For the healthy human being require to breath in a clean air but due to increasing the transportation system fresh air get polluted. Transport system make the impact on environment in which we live. Increase in vehicle gives rise to increasing traffic related pollutant emission. Therefore, to track the effect of this pollution on environment and health of individual it is necessary to track the level of pollution in urban and sub urban areas. Many health related issues are arising from air pollution. Major source of air pollution is road traffic emission which emits the $97 \%$ of $\mathrm{CO}$ and $75 \%$ of NO. Therefore, air quality monitoring is needed in order to provide useful information about the pollution and can take appropriate measures to mitigate the negative impact whenever it is necessary. The purpose of monitoring the air quality is not only to collect the data but also provide the information which is required by the scientist, planners, policy makers to make a decision on improving and managing the environment [1]. The main mission of air quality monitoring network is to record the concentration of pollution and other parameter related to the pollution and deliver these information or data to the population to warn against the any danger.

\section{NEED OF MONITORING}

Clean air is vital need for every human being. Polluted air causes many health problems and several damages. Therefore to make any step ahead of controlling the pollution rate it is necessary to monitor the air quality which may help us to make a right decision at right time. There are various causes of increasing the pollution such as smoke automobile exhaust, chemical discharge from industries, radioactive substance etc.these are main reason of decreasing the quality of air. The main gases which directly affect the human health are carbon monoxide (CO), hydrogen sulphide, sulphur dioxide (SO2),Nitrogen dioxide(NO2) and the main contribution of these gases are traffic related pollutant emission. Huge efforts are required to improve the quality of air in both outdoor and indoor environment. Monitoring of environment has been controlled from manual to the automatic control step by step. There are various improvement in the instrument of environment monitoring but still cannot meet the harsh environment [2].

\section{A. Technique used for pollution monitoring}

Previously the air pollution monitoring is done via computerized tomography technique which generate a two dimensional map of pollutant concentration. It provides a many advantages over the differential absorption method. In this system there is a single laser source located at the centre of the area. This laser beam is rotated and directed towards the circumference of the circle. There is a cylindrical mirror so that incident laser beam is reflected in a fan beam over angle across the circle. The beam from the mirrors is the circular region and strikes a set of detectors lie in same plane parallel to the ground. This technique focus on lower transmitted laser energy increasing the range and ability to monitor the area that contains several pollutant sources [3].

Another way of monitoring the air pollution is via the online GPRS sensors array which has been designed, implemented and tested. This system unit that consists at a single chip of microcontroller and a pollution server which is a high end personal application server with a internet connectivity where the mobile data acquisition unit that collect the pollution level \& pack it into a frame with GPS location, date and time. This frame is uploaded to the GPRS modem and transmitted to the pollution server via the public mobile network. A data base server which is attached to the pollution level which is used by the various client. Pollution server for storing the pollution level which is used by the various clients. Pollution server having a interfaced with the Google map to provide a real time pollutants level as well as the location in large metropolitan area [4]. 


\section{B. Wireless sensor network for real time monitoring}

A distributed infrastructure consists of a wireless sensor network and grid computing technology for air pollution monitoring as well as mining. However, the two layer network architecture and peer to peer e-science grid architecture and distributed data mining algorithms are used in order to collect the data and tiny operating system is used to examine the operation and performance of the wireless sensor network [5].Wireless sensor network is the great achievement in this field.

An effective solution for the pollution monitoring using a wireless sensor network to provide a real time pollution data. The various gases like $\mathrm{CO} 2, \mathrm{NO} 2$ are calibrated by using a appropriate calibration technologies and these precalibrated sensors are integrated with the wireless sensor using a multi hop data aggregation algorithm. A light weight middleware and web interface in order to view the one pollution data in the form of charts and number. It is also available on the internet. The other parameters like temperature and humidity are also sensed along with the gas concentrations which enable the data analysis through the data fusion techniques this system provide accurate pollutant data [6].

The air quality monitoring system combines with the virtual instrument technology \& frequency hopping communication technology to achieve the wireless data transmission. By using a spectrum hole detection specimens that adjust a carrier frequency according to the result $\&$ made a full use of available radio spectrum with this specimen there is no signal interference during the wireless transmission process $\&$ the system can receive the real time information effectively and the gas concentration can show clearly and easy to read by the non professional staff also [7].

The air quality monitoring station are used to monitor the quality of air but most of this method are expensive and provide a low resolution sensing data and these stations are less densely deployed therefore the system consist of sensor mode gateway and back end platform controlled by the lab view program through which the data can be stored in the database the system deployed to the main road in the city to monitor the carbon monoxide concentration caused by the vehicle emission the advantages of these wireless sensor network is that it is easy to set up, inexpensive and also provide a real time data[8].

The system in which several monitoring station communicate wirelessly with the backend server using machine to machine communication \& each station equipped with the metro logical sensor and gaseous sensor for data logging and wireless communication capabilities. The backend server collects the real time data from station and converts it in to the information which is used by the user through the web portals and mobile application [9].

The small scale of wireless sensor station to communicate with the backend server and provide their measurement in a real time however the collected data are process and analyse in order to provide these data in different format to the end user [10].

\section{Centralized monitoring}

Different sensors are deploying to the different region and each sensor must send their collected information to server so that the end user can easily see the pollution information in the different area. Centralize monitoring ensure the quality, improve the ability and integrity of data. Collected data are uploaded to the cloud dataset so that it can be analyze or viewed for future use. All these uploaded data are managed in database management system over the centralize database with this available information the user can search the record as per their requirement.

\section{Pollution level monitor over the google map}

The main objective of monitoring is to display the collected information in user friendly format. The mobile application and websites are developing in order to display the real time data that contains previous history and recent measurement of pollution level. Only the authorized user can access the website which is easily available to the public when the permission is granted. Website allows displaying the different level of pollution in different area over the Google map. with the help of internet connectivity it is possible to display the different level of pollution at different area on the Google map [11].

\section{E. Types of sensor}

There are different types of sensors are available for collecting the atmospherically data. Such as Temperature sensor, Humidity sensor, Rain sensor, Gas Sensor etc...different types of gas sensors are available to collect the different gases from the road traffic emission such as $\mathrm{CO} 2$ sensor,NO2 sensor,SO2 sensor etc. Wireless sensor network built a node where each node is connected to one sensor. With the additional sensors may help to enhance the network and monitoring the additional pollutants. With the help of sensors it may possible to collect the environment related information. It is deployed in several cities to monitor the concentration of dangerous gases for citizen. Air quality measurement can process and presented in a real time to the end user in a friendly format to spread environmental awareness among the population and allow taking appropriate precaution when it is needed.

\section{IV.CONCLUSION}

To monitor the air pollution with the wireless sensor network has several benefits over the traditional environment. Wireless sensor network has its own advantage such as low cost, easy to setup and provide a real time pollutant data. Monitoring stations which are used to analyze and collect the real time pollutant data from the road traffic emission. To monitor the pollution level from different area of glance is a difficult task and it requires a large infrastructure setup and proper management but if system can segment the pollution level as per the area so it can be better monitored and better solution can be provided. In future, the system can also implement the web based monitoring of the air pollution 
level and Google map which is used for live map view of pollution level. Accurate reading of pollution level is important in order to provide a guidance for the people who suffer from the asthmatic problem with this information they may help to choose the alternate healthy route.

\section{REFERENCES}

[1] Abdullah Kadri, Elias Yaacoub, Mohammed Mushtaha, And Adnan Abu-Dayya "Wireless Sensor Network For Real-Time Air Pollution Monitoring" IEEE Forum On Strategic Technology -2013.

[2] Haibao Wang, Tingting Wu ,And Guangjie Wu,"Air Quality Monitoring System Based On Frequency Hopping System 2010 IEEE.

[3] Robert L.Byer,Lawrence A.Shepp, "Two-Dimensional Remote AirPollution Monitoring Viatomography".Vol.4/ March 1979 / OPTICS LETTERS.

[4] Yajie Ma,Mark Richards,Moustafa Ghanem,Yike Guo,And John Hassard,"Air Pollution Monitoring And Mining Based On Sensor Grid In London"Sensor 2008.

[5] Al-Ali, A. R.; Zualkernan, I.; Aloul, F., "A Mobile GPRS Sensors Array For Air Pollution Monitoring," Sensors Journal, IEEE, Vol.10, No.10, Pp.1666, 1671, Oct. 2010.

[6] Raja Vara Prasad Y1, Mirza Sami Baig2, Rahul K. Mishra3, P. Rajalakshmi4, U. B. Desai5 And S.N. Merchant6" "Real Time Wireless Air Pollution Monitoring System" Ictact Journal On Communication Technology: Special Issue On Next Generation Wireless Networks And Applications, June 2011, Volume - 2, Issue - 2.

[7] Haibao Wang, Tingting Wu , And Guangjie Wu, "Air Quality Monitoring System Based On Frequency Hopping System" International Conference On Intelligent Control And Information Processing August 2010.

[8] Jen-Hao Liu, Yu-Fan Chen, Tzu-Shiang Lin, And Da-Wei Lai ,Tzai-Hung Wen, Chih-Hong Sun, And Jehn-Yih Juang,Joe-Air Jiangdeveloped Urban Air Quality Monitoring System Based On Wireless Sensor Networks 2011 Ieee.

[9] Srinivas Devarakonda, Parveen Sevusu, Hongz Hang Liu, Ruilin Liu, Liviu Iftode, Badri Nath Urbcomp' Real-Time Air Quality Monitoring Through Mobile Sensing In Metropolitan Areas'13, August $2013 \mathrm{Acm}$.

[10] Fouzi Harrou, Mohamed Nounou,Hazem Nounou "Detecting Abnormal Ozone Levels Using Pca Based Glr Hypothesis Testing”2013 Ieee Symposium On Computational Intelligence And Data Mining.

[11] Elias Yaacoub,Abdullah Kadri,Mohammad Mushtaha,And Adman Abu-Dayya,"Air Quality Monitoring And Analysis In Qatar Using A Wireless Sensor Network Deployment"596-601,2013 Ieee.

\section{BIOGRAPHIES}

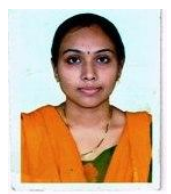

Snehal V Sirsikar received undergraduate degree in Electronics Engineering in the year of 2009.She is currently student of ME in Wireless communication and Computing branch from Priyadarshini college of

engineering.

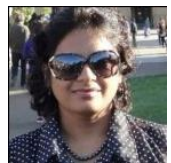

Mrs.Priya Karemore obtained M.Tech master degree in the year of 2007. She has 12 year of teaching experience. She Is currently working as a assistant professor at Priyadarshini college of engineering . 